\title{
The Initialization Of Basal Sliding Coefficients For Antarctica A Lyapunov Based Approach
}

\author{
Firas Mourad $^{1}$, Emmanuel Witrant $^{1}$ and Frank Pattyn ${ }^{2}$
}

\begin{abstract}
Models describing natural phenomena can depend on parameters that cannot be directly measured, hence the necessity to develop inverse techniques to determine them. The goal of this paper is to utilize such a technique to enable better initialization of ice sheet models for Antarctica. This will enable models to produce better forecasts as part of climate studies. The parameter of interest is the basal sliding coefficient, which characterizes the contact of the ice sheet with the bed underneath. A Lyapunov based approach is proposed to control the convergence of the 1D inhomogeneous transport model toward a feasible equilibrium matching the measurements. This method results in a new update law for the coefficient inversion. The results, which show an improved convergence toward the observed ice thickness, are compared with a currently used inverse method.
\end{abstract}

\section{INTRODUCTION}

Antarctica has been the subject of numerous studies as it encompasses a large variety of phenomena that impact reality around us. The understanding and study of past and future behavior of the Antarctic ice sheet requires a good initialization of the models used to simulate the dynamics of the ice. Initializing such models with the observed surface topography and velocity field requires the knowledge of basal characteristics of the ice sheet. Such characteristics cannot be directly measured. In this paper, the focus is on the basal sliding coefficient $A_{s}$ that describes the sliding of ice sheets over the basal bed. Using a constant $A_{s}$ in ice sheet models forward in time leads to a simulated ice sheet that is not necessarily in close agreement with the observed one. Alternatively, spatial variations of the basal sliding coefficients can be obtained through an iterative method, thus guaranteeing a simulated ice sheet close to the observation. Our method utilizes the misfit between the simulated and observed ice thickness in order to modify $A_{s}$. It will be compared to that of Pollard and DeConto [1] as their method also uses such a misfit to iteratively update the basal sliding coefficient. This parameter can be constrained by noticing that it acts as a transport coefficient for the ice thickness $h$, a quantity whose space distribution is measured for Antarctica. The evolution of $h$ can be modeled by a diffusive nonlinear partial differential equation (PDE) [2]. This fact is exploited here using a Lyapunov-based technique to find the distribution of $A_{s}$ that ensures the stability and exponential convergence of the modeled $h$ toward its measured value. In fact, this

\footnotetext{
${ }^{1}$ Firas Mourad and Emmanuel Witrant are at Université Grenoble Alpes, CNRS, GIPSA-lab, Department of Control, F-38000 Grenoble, France firas.mouradegipsa-lab.grenoble-inp.fr, emmanuel.witranteuniv-grenoble-alpes. fr

${ }^{2}$ Frank Pattyn is at Université Libre de Bruxelles, Laboratoire de Glaciologie, B-1050, Brussels, Belgium fpattyn@ulb.ac.be
}

convergence analysis was first introduced by A. M. Lyapunov in 1892 [3] when studying the properties of motion, which is of course described by PDEs. Since then, the Lyapunov method has been widely used, e.g. see [4], [5] and [6], and has not only included motion but a diverse array of phenomena [7], [8].

Ice-sheet models can vary depending on their complexity, from models solving the full Stokes equations [9] to simpler models where the Shallow-Ice approximation (SIA) [10], the Shallow-Shelf approximation (SSA) [11], or a combination of the two [12] is used. Such models can also vary in the number of spatial dimensions considered, from spatially distributed depth-dependent 3D models [9] to depth-integrated 1D models [13]. In this paper we propose a first proof of concept based on the one dimensional case, and as we are interested in the ice in contact with the bedrock we decided to investigate models with SIA. This approximation does not include sliding, as the ice is considered to be frozen to the bedrock. In order to induce sliding a sliding law can be added by either choosing a nonlinear Weertman sliding law [14] or a linear Coulomb friction law [15]; we decided to use the former. We thus propose a law based on Lyapunov analysis that will pave the way to extend the study to hybrid and two dimensional ice-sheet models, which will in turn enable the initialization of models like the fast Elementary Thermomechanical Ice Sheet model (f.ETISh) [16].

It is worthy to mention that our approach does not imply finding a Lyapunov function that will prove convergence relative to a given control law. In fact, the reverse is done as a simple Lyapunov function is chosen and an appropriate update law is calculated in order to fulfill the required conditions for convergence.

The performance of the proposed method is compared with the one of Pollard and DeConto [1] in terms of cumulative absolute error, difference between the simulated and observed ice thickness, convergence time and the estimated $A_{s}$. The robustness with respect to the initial value of basal sliding coefficient $A_{s 0}$ is also investigated.

This paper is organized as follows: in Section 2, the one dimensional nonlinear ice diffusion equation is introduced and simplified under SIA assumptions. Section 3 shows the linearization of the PDE. In Section 4, Lyapunov analysis is carried out and an appropriate update law guaranteeing convergence is chosen. Section 5 presents the simulations carried out on the linear and nonlinear systems and the obtained results are discussed. 
TABLE I

SYSTEM VARIABLES.

\begin{tabular}{|c|c|c|}
\hline Symbol & Description & Units \\
\hline$\dot{a}$ & Surface mass balance & $\mathrm{ma}^{-1}$ \\
\hline$A$ & Temperature-dependent coefficient & $\mathrm{Pa}^{-3} a^{-1}$ \\
\hline$A_{s}$ & Basal sliding coefficient & $\mathrm{ma}^{-1} \mathrm{~Pa}^{-1}$ \\
\hline$A_{s 0}$ & Initial value of basal sliding coefficient & $\mathrm{ma}^{-1} \mathrm{~Pa}^{-1}$ \\
\hline $\bar{A}_{s}$ & Observed basal sliding coefficient & $\mathrm{ma}^{-1} \mathrm{~Pa}^{-1}$ \\
\hline$b$ & Bedrock elevation & $m$ \\
\hline$d$ & Diffusion coefficient & $\mathrm{m}^{2} \mathrm{a}^{-1}$ \\
\hline$g$ & Gravitational acceleration & $\mathrm{m} \mathrm{s}^{-2}$ \\
\hline$h_{0}$ & Initial value of ice thickness & $\mathrm{m}$ \\
\hline$h$ & Ice thickness & $\mathrm{m}$ \\
\hline $\bar{h}$ & Observed ice thickness & $\mathrm{m}$ \\
\hline$t$ & Time & $\mathrm{m}$ \\
\hline$x$ & Spacial variable & $\mathrm{ma}$ \\
\hline $\bar{u}$ & Vertical mean horizontal velocity & $\mathrm{ma}$ \\
\hline$v_{b}$ & Basal sliding velocity & $\mathrm{m}$ \\
\hline$\Delta h$ & Error on the observed ice thickness & $\mathrm{Pa}$ \\
\hline$\rho$ & Ice density & $\mathrm{ma}^{-3}$ \\
\hline$\tau_{b}$ & Basal drag & \\
\hline$\tau_{d}$ & Driving stress & \\
\hline & & \\
\hline
\end{tabular}

\section{REFERENCE MODEL AND PROBLEM FORMULATION}

The one-dimensional evolution of ice thickness is described by the following diffusive PDE:

$$
h_{t}=\left(d(h+b)_{x}\right)_{x}+\dot{a}
$$

Where $h$ is the ice thickness, $b$ the bedrock elevation, $d$ the diffusion coefficient and $\dot{a}$ the surface mass balance. All partial derivatives are denoted by $t$ and $x$ subscripts; e.g. $\frac{\partial f}{\partial x}=f_{x}$. SIA enables the use of the following set of relationships:

$$
\left\{\begin{array}{l}
d=-\bar{u} h(h+b)_{x}^{-1} \\
\bar{u}=v_{b}+\frac{2 . A}{5} h \tau_{d}^{3} \\
v_{b}=A_{s} \tau_{b}\left|\tau_{b}\right| \\
\tau_{b} \approx \tau_{d}=-\rho g h(h+b)_{x}
\end{array}\right.
$$

Where $A$ is a temperature-dependent coefficient, $g$ the gravitational acceleration, $\bar{u}$ the vertical mean horizontal velocity, $v_{b}$ the basal sliding velocity, $\rho$ the ice density, $\tau_{b}$ the basal drag and $\tau_{d}$ the driving stress. A list of variables can be found in Table I.

Substituting (2) into (1) gives:

$$
\left\{\begin{array}{l}
h_{t}=\left(\rho^{2} g^{2} A_{s} h^{3}(h+b)_{x}\left|(h+b)_{x}\right|\right)_{x} \\
+\left(\frac{2}{5} A \rho^{3} g^{3} h^{5}(h+b)_{x}^{3}\right)_{x}+\dot{a} \\
\forall \in\left[x_{1}, x_{2}\right] \text { and } t \in[0, T] \\
h\left(x_{1}, t\right)=\bar{h}\left(x_{1}\right) \text { and } h\left(x_{2}, t\right)=\bar{h}\left(x_{2}\right) \\
h(x, 0)=\bar{h}(x)+\Delta h
\end{array}\right.
$$

where $\Delta h$ is some error on the observed ice thickness $\bar{h}$. Our estimation problem is formulated as finding the distributed profile $A_{s}(x)$ such that the solution of (3) converges toward $\bar{h}$ (which is supposed to be an equilibrium). The nonlinear nature of (3) clearly does not allow us to find an analytical solution directly and we propose to use $A_{s}$ as a feedback gain that stabilizes the linearized dynamics through an iterative method.

\section{LINEARIZED DYNAMICS}

Linearizing the dynamics around an equilibrium point is a common practice when dealing with PDEs. A first order Taylor series expansion around the equilibrium $h=\bar{h}$ and $A_{s}=\overline{A_{s}}$ is carried out. A similar approach was done for another diffusive system in [17]. As previously stated, the aim of this paper is to find $\overline{A_{s}}$. Expanding (3) gives:

$$
\begin{aligned}
h_{t} & =f\left(A_{s}, A_{s_{x}}, h, h_{x}, h_{x x}\right)=\rho^{2} g^{2}\left(A_{s x} h^{3}(h+b)_{x}\left|(h+b)_{x}\right|\right. \\
& \left.+3 A_{s} h_{x} h^{2}(h+b)_{x}\left|(h+b)_{x}\right|+2 A_{s} h^{3}(h+b)_{x x}\left|(h+b)_{x}\right|\right) \\
& +\frac{2}{5} \rho^{3} g^{3} A\left(5 h_{x} h^{4}(h+b)_{x}^{3}+3 h^{5}(h+b)_{x x}(h+b)_{x}^{2}\right)+\dot{a}
\end{aligned}
$$

The linearization is carried out as follows:

$$
\begin{aligned}
h_{t} & =\bar{f}+\left(A_{s}-\overline{A_{s}}\right) \frac{\partial \bar{f}}{\partial A_{s}}+\left(A_{s x}-\overline{A_{s x}}\right) \frac{\partial \bar{f}}{\partial A_{s x}}+(h-\bar{h}) \frac{\partial \bar{f}}{\partial h} \\
& +\left(h_{x}-\bar{h}_{x}\right) \frac{\partial \bar{f}}{\partial h_{x}}+\left(h_{x x}-\bar{h}_{x x}\right) \frac{\partial \bar{f}}{\partial h_{x x}}+\dot{a}
\end{aligned}
$$

where $\bar{f}=f\left(\overline{A_{s}}, \overline{A_{s x}}, \bar{h}, \bar{h}_{x}, \bar{h}_{x x}\right)$.

Denoting $\tilde{A}_{s}=A_{s}-\overline{A_{s}}, \tilde{A}_{s_{x}}=A_{s x}-\overline{A_{s x}}, \tilde{h}=h-\bar{h}, \tilde{h}_{x}=h_{x}-\bar{h}_{x}$ and $\tilde{h}_{x x}=h_{x x}-\bar{h}_{x x}$, and noticing that $\tilde{h}_{t}=h_{t}$ (due to the fact that $\bar{h}_{t}=0$ as it represents the equilibrium of the system) we obtain the linearized dynamics as:

$$
\tilde{h}_{t}=c_{2} \tilde{A}_{s}+c_{3} \tilde{A_{s x}}+c_{4} \tilde{h}+c_{5} \tilde{h}_{x}+c_{6} \tilde{h}_{x x}
$$

where the coefficients $c_{2}$ to $c_{6}$ are detailed in Appendix A. Note that $c_{1}=\bar{f}+\dot{a}=\bar{h}_{t}=0$.

The boundary and initial conditions become:

$$
\left\{\begin{array}{l}
\tilde{h}\left(x_{1}, t\right)=\tilde{h}\left(x_{2}, t\right)=0 \\
\tilde{h}(x, 0)=\Delta h
\end{array}\right.
$$

\section{LYAPUNOV ANALYSIS}

This section is divided into two parts: the first part deals with the choice of an appropriate Lyapunov function, while the second part focuses on finding $A_{s}$ which improves the system convergence.

\section{A. Lyapunov Function Candidate and its Derivative}

The Lyapunov function is often chosen to be an energylike function that needs to be dissipated with time. In the case of the system described by (6)-(7) a natural choice is:

$$
V=\frac{1}{2} \int_{x_{1}}^{x_{2}} \tilde{h}^{2} d x
$$

Theorem 1: The time derivative $V_{t}$ of the function $V$ given by (8) verifies:

$$
V_{t}=\int_{x_{1}}^{x_{2}} \tilde{h}\left(c_{3} \tilde{A_{s}}\right)_{x} d x+\frac{1}{2} \int_{x_{1}}^{x_{2}} \tilde{h}^{2} c_{4} d x-\int_{x_{1}}^{x_{2}} c_{6} \tilde{h}_{x}^{2} d x
$$

$\forall t \in[0, T]$ along the solutions of (6)-(7) with the transport coefficients given in Appendix A. 
Proof: Differentiating (8) with respect to time gives:

$$
V_{t}=\int_{x_{1}}^{x_{2}} \tilde{h} h_{t} d x=T_{1}+T_{2}+T_{3}+T_{4}
$$

where:

$$
\left\{\begin{array}{l}
T_{1}=\int_{x_{1}}^{x_{2}} \tilde{h}\left(c_{2} \tilde{A}_{s}+c_{3} \tilde{A}_{s x}\right) d x \\
T_{2}=\int_{x_{1}}^{x_{2}} c_{4} \tilde{h}^{2} d x \\
T_{3}=\int_{x_{1}}^{x_{2}} c_{5} \tilde{h} \tilde{h}_{x} d x \\
T_{4}=\int_{x_{1}}^{x_{2}} c_{6} \tilde{h} \tilde{h}_{x x} d x
\end{array}\right.
$$

Integration by parts is performed on $T_{3}$ and $T_{4}$ to allow $\tilde{h}^{2}$ to appear inside the integrals. First, $T_{3}$ becomes:

$$
T_{3}=\int_{x_{1}}^{x_{2}} c_{5} \tilde{h} \tilde{h}_{x} d x=-\frac{1}{2} \int_{x_{1}}^{x_{2}} c_{5_{x}} \tilde{h}^{2} d x
$$

Notice that due to the boundary conditions, terms like $\left.\left(c_{5} \bar{h}^{2}\right)\right|_{x_{1}} ^{x_{2}}=0$. The same applies to all subsequent integrations. Then, $T_{4}$ can be expressed as:

$$
T_{4}=\int_{x_{1}}^{x_{2}} c_{6} \tilde{h} \tilde{h}_{x x} d x=\int_{x_{1}}^{x_{2}}\left(\frac{1}{2} c_{6_{x x}} \tilde{h}^{2}-c_{6} \tilde{h}_{x}^{2}\right) d x
$$

Thus, (10) can be written as:

$$
\begin{aligned}
V_{t} & =\int_{x_{1}}^{x_{2}} \tilde{h}\left(c_{2} \tilde{A}_{s}+c_{3} \tilde{A_{s x}}\right) d x-\int_{x_{1}}^{x_{2}} c_{6} \tilde{h}_{x}^{2} d x \\
& +\int_{x_{1}}^{x_{2}} \tilde{h}^{2}\left(c_{4}-\frac{1}{2} c_{5_{x}}+\frac{1}{2} c_{6_{x x}}\right) d x \\
& =\int_{x_{1}}^{x_{2}} \tilde{h}\left(c_{3} \tilde{A_{s}}\right)_{x} d x+\frac{1}{2} \int_{x_{1}}^{x_{2}} \tilde{h}^{2} c_{4} d x-\int_{x_{1}}^{x_{2}} c_{6} \tilde{h}_{x}^{2} d x
\end{aligned}
$$

where the last equality is obtained by noticing that $c_{4}-$ $\frac{1}{2} c_{5_{x}}+\frac{1}{2} c_{6_{x x}}=\frac{1}{2} c_{4}$ (see Appendix B for more details).

\section{B. Design of the Basal Sliding Coefficient}

Using the Lyapunov function discussed in the previous section, the convergence of (6)-(7) is assured with the following theorem:

Theorem 2: If there exists $\alpha$ and $\gamma>0$ such that:

$$
\frac{1}{2} c_{4}(x)(1-\alpha)-\gamma<0 \forall x \in\left[x_{1}, x_{2}\right]
$$

then choosing:

$$
\tilde{A_{s}}=-\frac{1}{c_{3}} \tilde{h} \int_{x_{1}}^{x} c_{4}(l) d l
$$

guarantees the exponential convergence of (6)-(7) and:

$$
\int_{x_{1}}^{x_{2}} \tilde{h}(x, t) d x \leq \int_{x_{1}}^{x_{2}} \tilde{h}(x, 0) d x e^{-\gamma t}
$$

Proof: Let $\tilde{A}_{s}$ as in (16) and replacing it in (9) gives:

$$
\begin{aligned}
V_{t} & =-\int_{x_{1}}^{x_{2}}\left(\tilde{h}^{2} c_{4}+\tilde{h} \tilde{h}_{x} \int_{x_{1}}^{x} c_{4}(l) d l\right) d x \\
& +\frac{1}{2} \int_{x_{1}}^{x_{2}} \tilde{h}^{2} c_{4} d x-\int_{x_{1}}^{x_{2}} c_{6} \tilde{h}_{x}^{2} d x
\end{aligned}
$$

Using integration by parts, the first integral becomes:

$$
-\int_{x_{1}}^{x_{2}}\left(\tilde{h}^{2} c_{4}+\tilde{h} \tilde{h}_{x} \int_{x_{1}}^{x} c_{4}(l) d l\right) d x=-\frac{1}{2} \int_{x_{1}}^{x_{2}} \tilde{h}^{2} c_{4} d x
$$

Replacing (19) in (18) gives:

$$
V_{t}=-\int_{x_{1}}^{x_{2}} c_{6} \tilde{h}_{x}^{2} d x
$$

Notice that $c_{6}(x) \geq 0$ from (27) in Appendix A. This enables the use of Wirtinger's inequality [18] on the above integral:

$$
-\int_{x_{1}}^{x_{2}} c_{6} \tilde{h}_{x}^{2} d x \leq-\frac{c_{\sigma_{\min }}}{C} \int_{x_{1}}^{x_{2}} \tilde{h}^{2} d x
$$

where $c_{6_{\min }}$ is the minimum of $c_{6}(x)$ and $C=\frac{\left(x_{2}-x_{1}\right)^{2}}{\pi^{2}}$. We now have:

$$
V_{t} \leq-\gamma \int_{x_{1}}^{x_{2}} \tilde{h}^{2} d x
$$

This concludes the proof as we have found:

$$
\alpha=1 \text { and } \gamma=\frac{c_{6_{\min }}}{C}=\frac{c_{6_{\min }} \pi^{2}}{\left(x_{2}-x_{1}\right)^{2}}
$$

\section{METHOD EVALUATION ON SIMULATIONS AND MEASUREMENTS}

\section{A. Iterative Calculations of $A_{s}$}

The results of Theorem 2 are used to obtain an iterative calculation of $A_{s}$ as follows. Notice that $\int_{x_{1}}^{x_{2}} c_{4}(l) d l$ is actually $c_{7}$ (Appendix B), which when divided by $c_{3}$ reduces to:

$$
\frac{1}{c_{3}} \int_{x_{1}}^{x_{2}} c_{4}(l) d l=\frac{3}{\bar{h}} \overline{A_{s}}+2 A \rho g \bar{h}\left|(\bar{h}+b)_{x}\right|
$$

Equation (16) thus becomes:

$$
\tilde{A_{s}}=-\tilde{h}\left(\frac{3}{\bar{h}} \overline{A_{s}}+2 A \rho g \bar{h}\left|(\bar{h}+b)_{x}\right|\right)
$$

Since $\overline{A_{s}}$ is our variable of interest and not known apriori, we consider an iterative update law to have the equilibrium state of the nonlinear model (3) converging toward $\bar{h}$. The equilibrium of the linearized dynamics (6)-(7) is thus updated toward a solution of the nonlinear dynamics (3). This is done with the following algorithm:

- Start with an initial guess of $A_{s}$ and initialize the nonlinear system with $h=\bar{h}$.

- Run the system with the last calculated $A_{s}$ to get close to an equilibrium (e.g. during a time period sufficiently large with respect to $\gamma$ ) and obtain $h$.

- At iteration $i$ calculate $\tilde{A}_{s}$ as in (25) and update $\overline{A_{s}}$ using:

$$
\left\{\begin{array}{l}
\overline{A_{s i+1}}=A_{s_{i}}-\tilde{A}_{s i} \forall x \in\left[x_{1}, x_{2}\right] \\
\text { if } \overline{A_{s i+1}}(x)<0 \text { then } \overline{A_{s i+1}}(x)=\overline{A_{s i}}(x) \\
\text { else if } \overline{A_{s i+1}}(x)<\overline{A_{s}} \text { min } \text { then } \overline{A_{s i+1}}(x)=\overline{A_{s}} \text { min } \\
\text { else if } \overline{A_{s i+1}}(x)>\overline{A_{s}} \text { max } \\
A_{s i+1}=\overline{A_{s i+1}}
\end{array}\right.
$$

- Stop the simulation after a predetermined maximum number of iterations or if $\int_{x_{1}}^{x_{2}}|\tilde{h}| d x \leq \epsilon$, where $\epsilon>0$ is an arbitrarily small scalar constant. 
Inverse problems can be ill posed as a unique solution might not exist [19]. It is a common practice to add a regularizing term in order to impose some degree of smoothness to the solution [20]. In our case we used a simple space averaging filter to smooth $\overline{A_{s}}$ after each update. This filtering also helps in rejecting high frequency variations and avoids numerical instabilities when solving for $h$. Also, $\overline{A_{s}}$ is kept bounded between $\overline{A_{\text {smin }}}=1 \times 10^{-10} \mathrm{ma}^{-1} \mathrm{~Pa}^{-2}$ and $\overline{A_{\text {smax }}}=$ $1 \times 10^{-5} \mathrm{~m} \mathrm{a}^{-1} \mathrm{~Pa}^{-2}$ which respectively represent the hard bedrock and the slipperiest deformable sediment.

\section{B. Simulation Results}

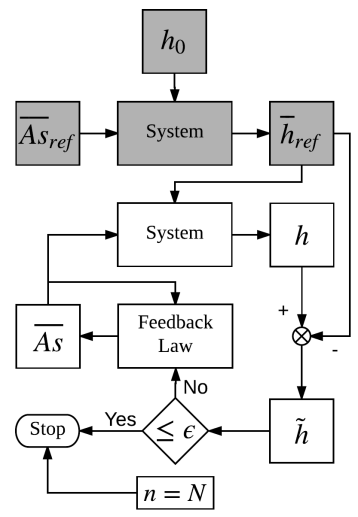

Fig. 1. Block diagram of the numerical experiment used to evaluate the estimation method on fictitious data. The blocks in gray represent phase 1 (synthesis of fictitious data), while those in white represent phase 2 (estimation). $n$ and $N$ are the iteration number and the maximum number of iterations, respectively.

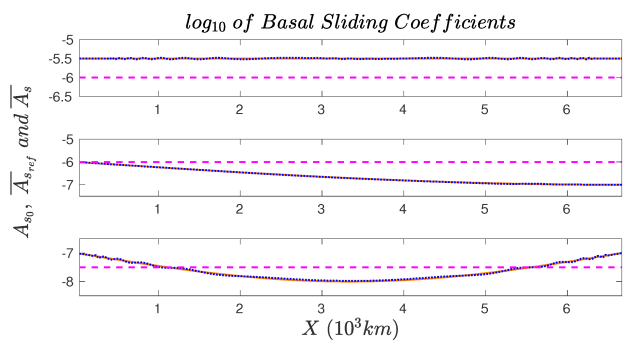

Fig. 2. Estimation for linear dynamics for three different profiles of $\overline{A_{s}}$ ref using fictitious data: comparison of $\overline{A_{s}}$ ref (full orange) and $\overline{A_{s}}$ (dotted blue). $\overline{A_{s}}$ (dashed purple) is the initial value of $A_{s}$.

The general set-up of the experiments consists of two phases. In the first phase a reference basal sliding coefficient $\overline{A_{\text {sref }}}$ is chosen and starting from an initial ice thickness $h_{0}$ the system is allowed to relax until it reaches steady state, thus giving us a reference ice thickness $\bar{h}_{\text {ref }}$ which will be treated as our observation. Then in phase two, $\bar{h}_{r e f},(25)$, and (26) are used to obtain $\overline{A_{s}}$ by updating the system after a fixed number of iterations. The simulations are stopped once a tolerance error is reached or after a predetermined number of iterations. This process is shown in Fig.1.

First, we evaluate our update law on the linearized model (6)-(7). The Crank-Nicolson method is used to discretize the

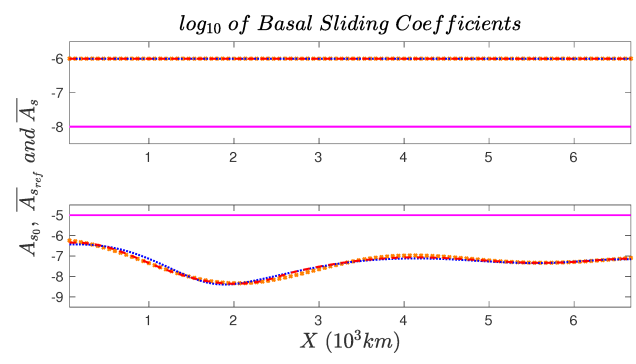

Fig. 3. Estimation of nonlinear dynamics, for two different profiles of $\overline{A_{\text {sref }}}$ using fictitious data: comparison of $\overline{A_{s}}$ ref (crossed orange) and $\overline{A_{s}}$ (dotted blue for Method 1 and dashed red for Method 2). $\overline{A_{s 0}}$ (dashed purple) is the initial value of $A_{s}$ for both methods.

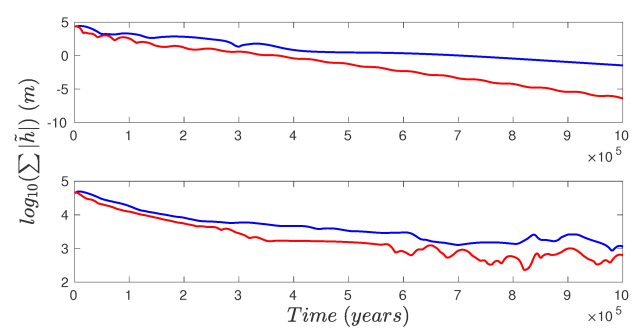

Fig. 4. Comparison of the evolution of the sum of the absolute error for Method 1 (blue) and Method 2 (red) for two different profiles of $\overline{A_{\text {sref }}}$ using fictitious data.

system. Here, we test the ability of the method to retrieve a chosen $\bar{A}_{\text {sref }}$. Figure 2 shows the results obtained for three different $\overline{A_{s}}$ ref. It is very clear that our feedback law manages to retrieve the reference in all three cases.

Second, the same is done for the nonlinear system and two update laws are tested: the method proposed by Pollard and DeConto and our newly proposed feedback law (defined as Method 1 and Method 2, respectively). During this test, a time step $d t=10$ years is used and the updates are done after every 100 iterations for both methods. Like in the previous case, $\overline{A_{\text {sref }}}$ is chosen and in Fig.3 we see that both methods are able to retrieve the reference. However, our method offers an improvement in the convergence rate and in the level of the error as is apparent in Fig.4.

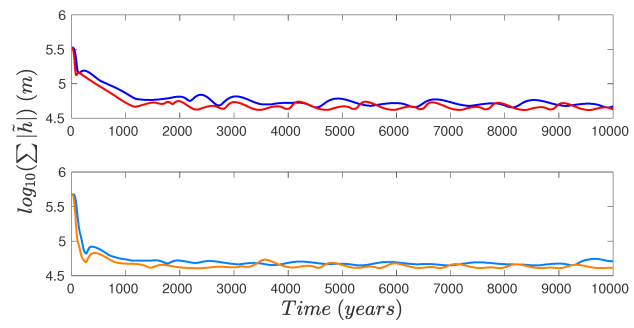

Fig. 5. Estimation of nonlinear dynamics, for two different profiles of $A_{s 0}$ using real data: comparison of the evolution of the sum of the absolute error Upper sub-plot: using $A_{s 0}=10^{-7} \mathrm{ma}^{-1} \mathrm{~Pa}^{-2}$, Method 1 in dark blue and Method 2 in red. Lower sub-plot: using $A_{s 0}=10^{-9} \mathrm{ma}^{-1} \mathrm{~Pa}^{-2}$, Method 1 in light blue and Method 2 in orange. 


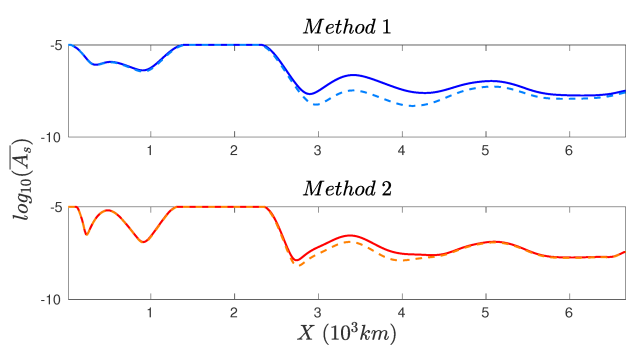

Fig. 6. Robustness of each method with respect to a change in $A_{s 0}$ using real data: upper sub-plot, for Method 1, in dark blue $A_{s 0}=10^{-7} \mathrm{ma}^{-1} \mathrm{~Pa}^{-2}$ and in dashed light blue $A_{s 0}=10^{-9} \mathrm{ma}^{-1} \mathrm{~Pa}^{-2}$. Lower sub-plot, for Method 2, in red $A_{s 0}=10^{-7} \mathrm{ma}^{-1} \mathrm{~Pa}^{-2}$ and in dashed orange $A_{s 0}=$ $10^{-9} \mathrm{ma}^{-1} \mathrm{~Pa}^{-2}$

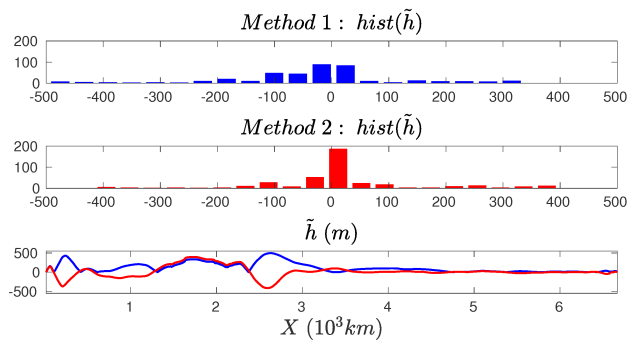

Fig. 7. Nonlinear system using real data, distribution of the error over a cross-section.

\section{Experimental Results}

Both methods are now evaluated on real data from the Bedmap2 dataset [21]. The chosen resolution is $10 \mathrm{~km}$, the time step is $d t=10$ years, updates are done after every 100 iterations, and the length of each simulation is 10,000 years. A standard Matlab solver (ode15s) is used to solve the nonlinear system described by (3) using the method of lines. The test focuses on the improvement in convergence and robustness in regards to $A_{s 0}$. Each method is tested with two different initial conditions $A_{s 0}\left(10^{-7}\right.$ and $\left.10^{-9} \mathrm{ma}^{-1} \mathrm{~Pa}^{-2}\right)$. In Fig. 5 we notice that Method 2 improves convergence in both cases, while producing less error. In Fig. 6 we see that Method 2 is more robust as the change in $A_{s 0}$ produces less variations in $\overline{A_{s}}$ compared to Method 1. Also, Method 2 seems to better capture the dynamics of the system, and variations in $A_{s}$ are more focused in the middle of the crosssection while being more consistent towards the boundaries. Next we look at the distribution of the error for both methods when $A_{s 0}=10^{-9} \mathrm{~m} \mathrm{a}^{-1} \mathrm{~Pa}^{-2}$. In Fig. 7 the histograms of both methods show that low-magnitude errors are more dominant for Method 2.

The last test is done by adding noise to $h_{r e f}$ and observing its effect on $\overline{A_{s}}$. Figure 8 shows that the addition of measurement noise will increase the cumulative error but without destabilizing the system, and that our estimate of $A_{s}$ does not deviate significantly from the one obtained from the noiseless data.

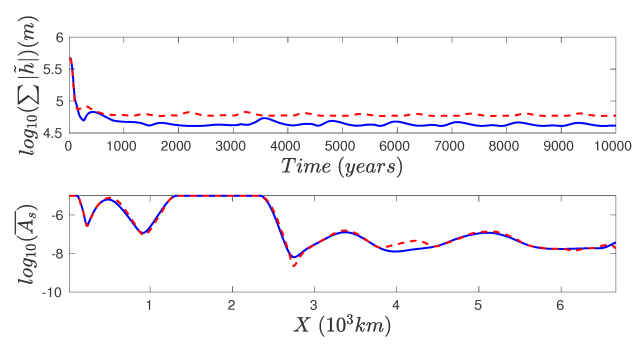

Fig. 8. Comparison of the evolution of the sum of the absolute error and the estimation of nonlinear dynamics for two different profiles of $\bar{h}_{\text {ref }}$ using real data (nominal in blue and noisy in dashed red).

\section{CONCLUSION AND FUTURE WORKS}

This paper proposes a new update law for the initialization of basal sliding coefficients in Antarctica. A Lyapunov-based approach is presented and an update law which guarantees the convergence toward the observed ice thickness is found. This law (25) is tested on both the linear (6)-(7) and nonlinear dynamics (3) of the one-dimensional evolution of ice thickness (1). Our method is tested against a currently used inverse technique [1]. The criteria of interest are the convergence rate, error levels and error distribution, as well as the robustness with respect to $A_{s 0}$. For the linear system, $\overline{A_{s} r e f}$ is retrieved as expected. For the nonlinear system, we found that our method converges toward $\bar{h}$ at an accelerated rate compared to Method 1. Also, our method produces an estimated $\overline{A_{s}}$ which captures more of the dynamics of the system, which consequently reduces the error between the simulated $h$ and measured $\bar{h}$. Also, our method proved to be more robust with respect to the initial guess of $A_{s 0}$.

Improvements on the proposed technique will be in the form of finding a Lyapunov function that can grant us control over the convergence rate toward $\bar{h}$. Seeking an adaptive algorithm with respect to the update steps could influence the convergence rate and improve robustness with respect to $A_{s 0}$. Also, a more sophisticated regularization method can improve our estimates.

These results pave the way for expanding this approach to include hybrid one-dimensional and two-dimensional icesheet models. This will enable a better initialization of such models which will in turn help understanding the past history and future behavior of ice sheets.

\section{ACKNOWLEDGMENT}

We would like to thank our colleagues at the Institut des Gosciences de l'Environement (IGE), Dr. Catherine Ritz and Dr. Olivier Gagliardini, for their valuable feedback and discussions. 


\section{APPENDIX A}

In this appendix, the full expressions for coefficients $c_{1}$ to $c_{6}$ are shown.

$$
\begin{aligned}
& c_{1}=\rho^{2} g^{2}\left(\overline{A_{s x}} \bar{h}^{3}(\bar{h}+b)_{x}\left|(\bar{h}+b)_{x}\right|+2{\overline{A_{s}}}^{3}(\bar{h}+b)_{x x}\left|(\bar{h}+b)_{x}\right|\right) \\
& +\frac{2 A}{5} \rho^{3} g^{3}\left(5 \bar{h}^{4} \bar{h}_{x}(\bar{h}+b)_{x}^{3}+3 \bar{h}^{5}(\bar{h}+b)_{x x}(\bar{h}+b)_{x}^{2}\right)+\dot{a} \\
& c_{2}=\rho^{2} g^{2}\left(3 \bar{h}_{x} \bar{h}^{2}(\bar{h}+b)_{x}\left|(\bar{h}+b)_{x}\right|+2 \bar{h}^{3}(\bar{h}+b)_{x x}\left|(\bar{h}+b)_{x}\right|\right) \\
& c_{3}=\rho^{2} g^{2} \bar{h}^{3}(\bar{h}+b)_{x}\left|(\bar{h}+b)_{x}\right| \\
& c_{4}=\rho^{2} g^{2}\left(3 \bar{A}_{s x} \bar{h}^{2}(\bar{h}+b)_{x}\left|(\bar{h}+b)_{x}\right|\right. \\
& \left.+6{\overline{A_{s}}}_{\bar{h}_{x}} \bar{h}(\bar{h}+b)_{x}\left|(\bar{h}+b)_{x}\right|+6{\overline{A_{s}}}^{2}(\bar{h}+b)_{x x}\left|(\bar{h}+b)_{x}\right|\right) \\
& +\frac{2 A}{5} \rho^{3} g^{3}\left(20 \bar{h}_{x} \bar{h}^{3}(\bar{h}+b)_{x}^{3}+15 \bar{h}^{4}(\bar{h}+b)_{x x}(\bar{h}+b)_{x}^{2}\right) \\
& c_{5}=\rho^{2} g^{2}\left(2{\overline{A_{s}}}_{\bar{h}} \bar{h}^{3}\left|(\bar{h}+b)_{x}\right|+6{\overline{A_{s}}}_{h_{x}} \bar{h}^{2}\left|(\bar{h}+b)_{x}\right|\right. \\
& \left.+2{\overline{A_{s}}}^{3}(\bar{h}+b)_{x x} \frac{\left|(\bar{h}+b)_{x}\right|}{(\bar{h}+b)_{x}}+3{\overline{A_{s}}}^{2}(\bar{h}+b)_{x}\left|(\bar{h}+b)_{x}\right|\right) \\
& +\frac{2 A}{5} \rho^{3} g^{3}\left(15 \bar{h}_{x} \bar{h}^{4}(\bar{h}+b)_{x}^{2}+6 \bar{h}^{5}(\bar{h}+b)_{x x}(\bar{h}+b)_{x}\right. \\
& \left.+5 \bar{h}^{4}(\bar{h}+b)_{x}^{3}\right) \\
& c_{6}=2 \rho^{2} g^{2} \bar{A}_{s}{ }^{3}\left|(\bar{h}+b)_{x}\right|+\frac{6 A}{5} \rho^{3} g^{3} \bar{h}^{5}(\bar{h}+b)_{x}^{2}
\end{aligned}
$$

\section{APPENDIX B}

Here, the simplifications done during the Lyapunv analysis are expanded. Recalling $c_{6}$ and differentiating with respect to $x$ gives:

$$
\begin{aligned}
c_{6_{x}} & =\rho^{2} g^{2}\left(2 \overline{A_{s x}} \bar{h}^{3}\left|(\bar{h}+b)_{x}\right|+6 \overline{A_{s} h_{x}} \bar{h}^{2}\left|(\bar{h}+b)_{x}\right|\right. \\
& \left.+2{\overline{A_{s}}}^{3}(\bar{h}+b)_{x x} \frac{\left|(\bar{h}+b)_{x}\right|}{(\bar{h}+b)_{x}}\right)+\frac{2 A}{5} \rho^{3} g^{3}\left(15 \bar{h}_{x} \bar{h}^{4}(\bar{h}+b)_{x}^{2}\right. \\
& \left.+6 \bar{h}^{5}(\bar{h}+b)_{x x}(\bar{h}+b)_{x}\right)
\end{aligned}
$$

Now, subtracting $c_{5}$ from (28) gives:

$$
\begin{aligned}
& c_{6_{x}}-c_{5}=\rho^{2} g^{2}\left(2 \overline{A_{s x}} \bar{h}^{3}\left|(\bar{h}+b)_{x}\right|+6 \overline{A_{s}} h_{x} \bar{h}^{2}\left|(\bar{h}+b)_{x}\right|\right. \\
& +2{\overline{A_{s}}}^{3}(\bar{h}+b)_{x x} \frac{\left|(\bar{h}+b)_{x}\right|}{(\bar{h}+b)_{x}}-2 \overline{A_{s x}} \bar{h}^{3}\left|(\bar{h}+b)_{x}\right| \\
& -6 \overline{A_{s}} \bar{h}_{x} \bar{h}^{2}\left|(\bar{h}+b)_{x}\right|-2 \overline{A_{s}} h^{3}(\bar{h}+b)_{x x} \frac{\left|(\bar{h}+b)_{x}\right|}{(\bar{h}+b)_{x}} \\
& \left.-3{\overline{A_{s}}}^{2}(\bar{h}+b)_{x}\left|(\bar{h}+b)_{x}\right|\right)+\frac{2 A}{5} \rho^{3} g^{3}\left(15 \bar{h}_{x} \bar{h}^{4}(\bar{h}+b)_{x}^{2}\right. \\
& +6 \bar{h}^{5}(\bar{h}+b)_{x x}(\bar{h}+b)_{x}-15 \bar{h}_{x} \bar{h}^{4}(\bar{h}+b)_{x}^{2} \\
& \left.-6 \bar{h}^{5}(\bar{h}+b)_{x x}(\bar{h}+b)_{x}-5 \bar{h}^{4}(\bar{h}+b)_{x}^{3}\right) \\
& =-3 \rho^{2} g^{2}{\overline{A_{s}}}^{2}(\bar{h}+b)_{x}\left|(\bar{h}+b)_{x}\right|-2 A \rho^{3} g^{3} \bar{h}^{4}(\bar{h}+b)_{x}^{3}
\end{aligned}
$$

For ease of notation, we define (27) as $c_{7}$. Thus, it was found that $c_{6_{x}}-c_{5}+c_{7}=0 \Rightarrow c_{6_{x x}}-c_{5_{x}}+c_{7_{x}}=0$. The next step is to find the value of $c_{7_{x}}$ :

$$
\begin{aligned}
c_{7_{x}} & =\rho^{2} g^{2}\left(3 \overline{A_{s x}} \bar{h}^{2}(\bar{h}+b)_{x}\left|(\bar{h}+b)_{x}\right|\right. \\
& \left.+6 \overline{A_{s} h_{x}} \bar{h}(\bar{h}+b)_{x}\left|(\bar{h}+b)_{x}\right|+6{\overline{A_{s}}}^{2}(\bar{h}+b)_{x x}\left|(\bar{h}+b)_{x}\right|\right) \\
& +\frac{2 A}{5} \rho^{3} g^{3}\left(20 \bar{h}_{x} \bar{h}^{3}(\bar{h}+b)_{x}^{3}+15 \bar{h}^{4}(\bar{h}+b)_{x x}(\bar{h}+b)_{x}^{2}\right)
\end{aligned}
$$

From the above, we found that $c_{7_{x}}=c_{4}$ which implies:

$$
\begin{gathered}
c_{4}-\frac{1}{2} c_{5_{x}}+\frac{1}{2} c_{6_{x x}}=\frac{1}{2} c_{4} \\
\text { REFERENCES }
\end{gathered}
$$

\section{REFERENCES}

[1] D. Pollard and R. M. DeConto, "A simple inverse method for the distribution of basal sliding coefficients under ice sheets, applied to Antarctica", The Cryosphere, vol. 6, pp. 953971, 2012.

[2] P. Huybrechts, "The Antarctic ice sheet and environmental change: a three-dimensional modelling Study", Berichte für Polar-forschung, vol. 99, pp. 1241, 1992.

[3] A. M. Lyapunov, "The General Problem of the Stability of Motion", International Journal of Control, vol. 3, pp. 531773, 1992.

[4] H. Khalil, Nonlinear systems, 3rd ed. Upper Saddle River, NJ, USA: Prentice Hall, 2002.

[5] S. Lyashevskiy and A. U. Meyer, "Control System Analysis and Design Upon the Lyapunov Method", Proceedings of the American Control Conference, Seattle, WA, USA, vol. 5, pp. 3219-3223, 1995.

[6] M. Krstic and A. Smyshlyaev, "Adaptive Boundary Control for Unstable Parabolic PDEs-Part I: Lyapunov Design", IEEE Transactions on Automatic Control, vol. 53, no. 7, pp. 15751591, 2008.

[7] F. Bribiesca Argomedo, C. Prieur, E. Witrant, and S. Brémond, "A Strict Control Lyapunov Function for a Diffusion Equation With TimeVarying Distributed Coefficients", IEEE Transactions on Automatic Control, vol. 58, no. 2, pp. 290303, 2013.

[8] S. Marx, E. Cerpa, C. Prieur, and V. Andrieu, "Global stabilization of a Korteweg-de Vries equation with a distributed control", SIAM Journal on Control and Optimization, vol. 55, no. 3, pp. 1452-1480, 2017.

[9] L. Favier, O. Gagliardini, G. Durand, T. Zwinger, "A threedimensional full Stokes model of the grounding line dynamics: effect of a pinning point beneath the ice shelf", The Cryosphere, vol. 6, pp. 101-112, 2012.

[10] K. Hutter, Theoretical Glaciology, Dordrecht, Netherlands: Kluwer Academic Publishers, 1983.

[11] M. Weis, R. Greve, K. Hutter, "The theory of shallow ice shelves", Continuum Mechanics and Thermodynamics, vol. 11, pp. 15-50, 1999.

[12] E. Bueler, J. Brown, "Shallow shelf approximation as a "sliding law" in a thermomechanically coupled ice sheet model", Journal of Geophysical Research, vol. 114, 2009.

[13] D. Partridge, M. J. Baines, "A moving mesh approach to an ice sheet model", Computers and Fluids, vol. 46, pp. 381-386, 2011.

[14] J. Weertman, "On the sliding of glaciers", Journal of Glaciology, vol 3, no. 21, pp. 3338, 1957.

[15] V. L. Popov, Contact mechanics and friction, New York, NY, USA: Springer, 2010.

[16] F. Pattyn, "Sea-level response to melting of Antarctic ice shelves on multi-centennial timescales with the fast Elementary Thermomechanical Ice Sheet model (f.ETISh v1.0)", The Cryosphere, vol. 11, pp. 1851-1878, 2017.

[17] B. Mavkov, E. Witrant, C. Prieur, "Distributed control of coupled inhomogeneous diffusion in Tokamak plasmas", IEEE Transactions on Control Systems Technology, vol. PP, no. 99, pp. 1-8, 2017.

[18] H. Dym and H. P. McKean, Fourier series and integrals, New York, NY, USA: Academic Press, 1972.

[19] H. W. Engel, M. Hanke, A. Neubauer, Regularization of inverse problems, Dordrecht, Netherlands: Kluwer Academic Publishers, 1996.

[20] N. Petra et al., "An inexact Gauss-Newton method for inversion of basal sliding and rheology parameters in a nonlinear Stokes ice sheet model", Journal of Glaciology, vol. 58, no. 211, pp. 889-903, 2012.

[21] P. Fretwel et al., "Bedmap2: Improved Ice Bed, Surface and Thickness Datasets for Antarctica", The Cryosphere, vol. 7, no. 1, pp. 375-393, 2013 